\title{
Complex Signal Constellations in Cumulants- Based AMC: Statistics and Performance
}

\author{
Marko S. Pajic, Mladen Veinovic, and Vladimir D. Orlic, Senior Member, IEEE
}

\begin{abstract}
In this paper various complex signal constellations are considered in the context of Automatic Modulation Classification (AMC) based on a higher-order normalized cumulants value. Most of the constellations have been addressed so far with fourth-order cumulants as AMC features only. The goal of this paper is to provide comparable values of sixth-order cumulants' statistics for complex signal constellations as well, while resolving ambiguity in constellation shapes addressed at the same time and directing towards the criterion for estimation of expected classification performance.
\end{abstract}

Keywords - Constellation, complex signals, AMC, cumulants, statistics.

\section{INTRODUCTION}

$\mathrm{S}$ TARTING from the publication of a simple, very low complexity, robust method based on fourth-order cumulants that can easily be applied in a hierarchical manner to classify various digital signaling formats [1], many authors worked on Automatic Modulation Classification (AMC) algorithms with normalized cumulants used as features of interest. While the number and sophistication of digital signaling systems increase over years, an emerging need for intelligent modems capable of quickly discriminating signal types remains crucial for cognitive radio, cooperative communications and massive Internet of Things (IoT) surrounding. Thus, the idea of using simple AMC schemes, robust and appropriate for practical implementation, makes cumulants being (still) a natural choice.

Apart from the fourth-order normalized cumulants, the sixth-order normalized cumulants [2,3] were mostly considered for this purpose, by many authors worldwide. While eighth-order cumulants have been also analyzed [4], simplicity and some practical advantages of sixth-order cumulants prevail [5]. In recent researches simple cumulant features are additionally supported with complex classifiers which may result in excellent performance, such as neural networks or deep learning methods, considered for various sets of digital signals' constellations.

Paper received May 12, 2021; revised October 10, 2021; accepted December 14, 2021. Date of publication December 29, 2021. The associate editor coordinating the review of this manuscript and approving it for publication was Prof. Zorica Nikolić.

This research is financed by Vlatacom Institute of High Technologies, Serbia.

Marko S. Pajic and Vladimir D. Orlic are with Vlatacom Institute of High Technologies, Bul. M. Milankovica 5, 11070 Belgrade, Serbia (e mail: marko.pajic@vlatacom.com,vladimir.orlic@vlatacom.com).

Mladen Veinovic is with Singidunum University, Danijelova 32, 11000 Belgrade, Serbia (e mail: mveinovic@singidunum.ac.rs).
Still, many signal constellations were observed in early days of cumulant-based AMC research only, under only one of higher-order cumulant values. At the same time, in literature some discrepancies between the reported cumulant values of the same constellations can be found, coming mostly from the fact that authors didn't consider exactly the same constellation structures. The goal of work presented in this paper is to provide an overview of both fourth and sixth-order cumulant values of various signals constellations in one place, along with their statistical properties relevant for practical performance in AMC applications. While some of the constellations presented in this work are analyzed in the context of cumulants for the very first time, even for the most of other considered signal structures corresponding statistics are calculated and presented in this paper for the very first time, also.

The rest of the paper is organized as follows: in Section II cumulants' structures are defined, in Section III various complex signal constellations are discussed in AMC context, while classification performance is analyzed in Section IV, followed with concluding considerations.

\section{Normalized CUmUlantS For Signal Modulation CLASSIFICATION}

For a zero-mean random variable $x$, associated with a transmitted data sequence $x(n)$, the second-order cumulant $C_{21, x}=\operatorname{cum}\left(x, x^{*}\right)$ is given by:

$$
C_{21, x}=E\left(|x|^{2}\right) .
$$

The fourth-order cumulant $C_{42, x}=\operatorname{cum}\left(x, x, x^{*}, x^{*}\right)$ and the normalized fourth-order cumulant of the same variable are given as [1]:

$$
\begin{gathered}
C_{42, x}=E\left(\left|x^{4}\right|\right)-\left|E\left(x^{2}\right)\right|^{2}-2 E^{2}\left(\left|x^{2}\right|\right), \\
\hat{C}_{42, x}=C_{42, x} /\left(C_{21, x}\right)^{2} .
\end{gathered}
$$

The sixth-order cumulant $C_{63, x}=\operatorname{cum}\left(x, x, x, x^{*}, x^{*}, x^{*}\right)$ and the normalized sixth-order cumulant of the same random variable $x$ are derived as [3]:

$$
\begin{gathered}
C_{63, x}=E\left(|x|^{6}\right)-9 E\left(|x|^{4}\right) E\left(|x|^{2}\right)+ \\
+12\left|E\left(x^{2}\right)\right|^{2} E\left(|x|^{2}\right)+12 E^{3}\left(|x|^{2}\right), \\
\hat{C}_{63, x}=C_{63, x} /\left(C_{21, x}\right)^{3} .
\end{gathered}
$$

The calculation of cumulants of a received signal through eq. (1) - (5) in practice is executed via the calculation of mean-values over an ensemble of collected signal samples, which is implementable quite easily. If the number of samples is represented with $N$, the estimated values of 
normalized cumulants are always shaped with some portion of dispersion around expected (theoretical) values, proportionally to the value of $N$. This phenomenon was explored and described originally in [1], where the variance of the sample estimates of $C_{42, x}$ for complex constellations, with $N$ samples, is calculated as:

$N \operatorname{var}\left(C_{42, x}\right)=\left[m_{8,4}-m_{4,2}^{2}\right]+4 m_{2,1}\left[3 m_{4,2} m_{2,1}-2 m_{6,3}+2 m_{2,1}^{3}\right]$,

with $m_{k, m}=E\left[y^{k-m}\left(y^{*}\right)^{m}\right]$. For real constellations, the variance of the sample estimates of $C_{42, x}$ is given by:

$N \operatorname{var}\left(C_{42, x}\right)=\left[m_{8,4}-m_{4,2}^{2}\right]+6 m_{2,1}\left[5 m_{4,2} m_{2,1}-2 m_{6,3}+3 m_{2,1}^{3}\right]$.

A similar conclusion was reported for the sixth-order cumulants in [6], where the variance of $C_{63, x}$, for complex signals with $N$ samples, is given with:

$$
\begin{gathered}
N \operatorname{var}\left(C_{63, x}\right)=\left[m_{12,6}-m_{6,3}^{2}\right]+9\left[m _ { 2 , 1 } ^ { 2 } \left(48 m_{4,2} m_{2,1}^{2}-54 m_{4,2}^{2}\right.\right. \\
\left.+96 m_{2,1}^{4}-64 m_{6,3} m_{2,1}\right)+m_{4,2}\left(9 m_{4,2}^{2}+16 m_{6,3} m_{2,1},\right. \\
\left.\left.\quad-2 m_{8,4}\right)+m_{2,1}\left(17 m_{8,4} m_{2,1}-2 m_{10,5}\right)\right]
\end{gathered}
$$

while for real constellations, this variance is given by:

$$
\begin{aligned}
N \operatorname{var}( & \left.C_{63, x}\right)=\left[m_{12,6}-m_{6,3}^{2}\right]+9\left[m _ { 2 , 1 } ^ { 2 } \left(384 m_{4,2} m_{2,1}^{2}-126 m_{4,2}^{2}\right.\right. \\
& \left.+384 m_{2,1}^{4}-128 m_{6,3} m_{2,1}\right)+m_{4,2}\left(9 m_{4,2}^{2}+16 m_{6,3} m_{2,1}\right. \\
& \left.\left.-2 m_{8,4}\right)+m_{2,1}\left(25 m_{8,4} m_{2,1}-2 m_{10,5}\right)\right]
\end{aligned}
$$

Thus, reported error variances are directly proportional with sample size $N$ and take different values for different modulation formats.

While variances of the sample estimates of fourth-order cumulants for various Pulse Amplitude Modulation (PAM) constellations, along with a number of complex signal constellations were published in [1], variances of the sample estimates $C_{63, x}$ of PAM signals were published for the first time just recently [7]. In general, the sixth-order cumulants were mostly observed in the context of QPSK, V.29 and just a few rectangular QAM constellations [2,8], along with BPSK signals $[3,4]$, so variances of the sample estimates for many signal constellations remain published for $C_{42, x}$ only so far. It is of obvious interest to provide a comprehensive overview of various complex signal constellations' $C_{63, x}$ estimates and variances also, in one place. At the same time, it is of interest to resolve possible discrepancies in numerical results reported, appearing as a consequence of using even slightly different constellation shapes by different authors, which occur frequently in the case of analyzing 8-point V.29 and Quadrature Amplitude Modulation (QAM) constellations, for example, or star QAM-16 and 16-point V.29 as another example. Clear identification in both visual and numerical context of constellations analyzed via cumulant properties should be provided, since in many cases visual representation only shows to be insufficient for repeatability of reported results [9]. Moreover, there is a number of complex signal constellations which have not been analyzed in the context of higher - order cumulants at all. For all the reasons mentioned, we calculate and in Table 1 we provide the set of theoretic cumulant values and their corresponding variances, for various modulation constellations. Some specific complex constellations included in Table 1 are presented in Fig. 1 to Fig. 12, for additional precision in (both visual and numerical) identification.

\section{Complex Signal Constellations}

There are many complex signal constellations whose $\hat{C}_{63, x}$ value and corresponding variance is presented in Table 1 for the first time, starting from the standard rectangular QAM constellations (given with prefix " $r$ " in Table 1), [10]. To the best of our knowledge, this would be the case for all rectangular QAM constellations having more than 256 symbols. Well known rectangular QAM constellations are presented in Fig. 1, while a rectangular QAM-8 structure used for this analysis is given in Fig. 2.

TABLE 1: THEORETICAL CUMULANT STATISTICS FOR REAL AND COMPLEX CONSTELLATIONS, AND VARIANCES OF THEIR SAMPLE ESTIMATES

\begin{tabular}{l|c|c|c|c}
\hline Constellation & $\hat{C}_{63}$ & $N \operatorname{var}\left(C_{63, x}\right)$ & $\hat{C}_{42}$ & $N \operatorname{var}\left(C_{42, x}\right)$ \\
\hline BPSK & 16.0000 & 5040.00 & -2.0000 & 36.00 \\
PAM-4 & 12.1600 & 4612.00 & -1.3600 & 34.72 \\
PAM-8 & 11.7600 & 4455.00 & -1.2381 & 32.27 \\
PAM-16 & 11.6817 & 4419.70 & -1.2094 & 31.67 \\
PAM-32 & 11.6632 & 4411.00 & -1.2023 & 31.52 \\
PAM-64 & 11.6587 & 4409.00 & -1.2006 & 31.49 \\
QPSK & 4.0000 & 576.00 & -1.0000 & 12.00 \\
rQAM-8 & 3.3300 & 485.72 & -0.8900 & 11.11 \\
(8,8) QAM-16 & 2.6814 & 401.09 & -0.7800 & 10.24 \\
AMPM-8 & 2.5600 & 332.31 & -0.7200 & 9.54 \\
(12,4) QAM-16 & 2.5000 & 404.16 & -0.7711 & 10.74 \\
rQAM-32 & 2.1100 & 338.92 & -0.6900 & 9.70 \\
rQAM-16 & 2.0800 & 332.31 & -0.6800 & 9.54 \\
cQAM-8 & 2.0000 & 317.05 & -0.6670 & 9.33 \\
rQAM-128 & 1.9594 & 315.43 & -0.6573 & 9.31 \\
rQAM-512 & 1.9240 & 309.92 & -0.6494 & 9.21 \\
V29c [1] & 1.8400 & 298.00 & -0.6400 & 9.12 \\
rQAM-64 & 1.7970 & 289.38 & -0.6190 & 8.82 \\
rQAM-256 & 1.7345 & 279.84 & -0.6047 & 8.65 \\
rQAM-1024 & 1.7193 & 277.52 & -0.6012 & 8.61 \\
V29c (7200s) & 1.5700 & 266.59 & -0.5950 & 8.76 \\
V29 & 1.4897 & 262.45 & -0.5816 & 8.75 \\
(4,4,4,4) QAM-16 & 0.7380 & 161.98 & -0.4270 & 6.94 \\
sQAM-8 & 0.1600 & 116.92 & -0.3600 & 6.88 \\
sQAM-16 & 0.1600 & 116.92 & -0.3600 & 6.88 \\
\hline
\end{tabular}

Star QAM-8 [11] and star QAM-16 [12] constellations (given with prefix " $s$ " in Table 1) are analyzed for the first time in the context of cumulants also, and their structures are presented in Fig. 3 and Fig. 4, respectively.

Various circular QAM constellations, namely cQAM-8 (Table 1) with 8 symbols, or $(12,4),(8,8)[13]$ and $(4,4,4,4)$ [11] with 16 symbols, are analyzed in the context of (any) cumulants for the first time also. Their fourth and sixthorder cumulant statistics are presented for the first time in Table 1, to the best of our knowledge, while their structures are presented in Fig. 5 - Fig. 8, respectively.

While AMPM-8 constellation was considered under the fourth-order cumulants' statistics in [1], its sixth-order cumulant statistics, to the best of our knowledge, are given in Table 1 for the first time. The AMPM- 8 constellation structure used for this analysis is presented in Fig. 9, formed in correspondence with [14]. 


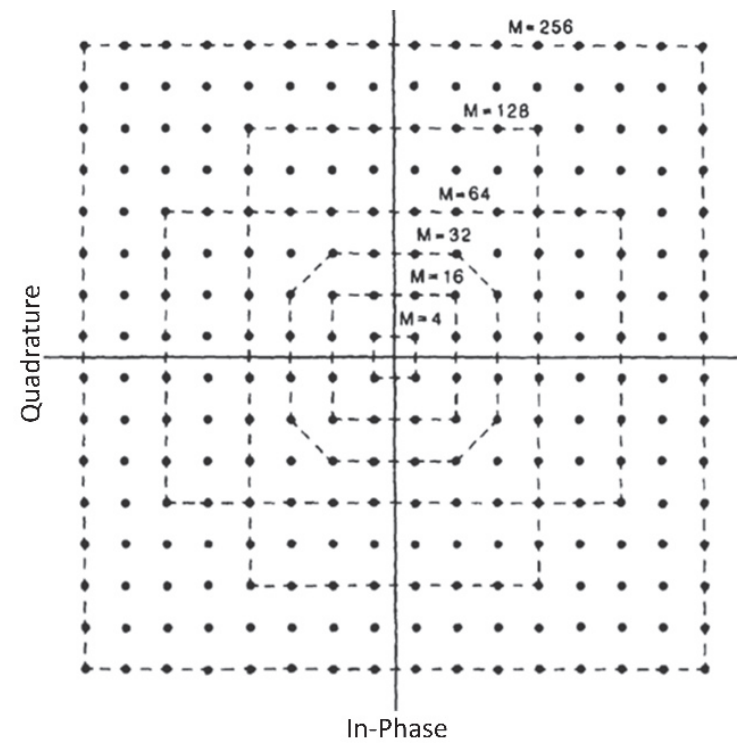

Fig. 1. Square QAM constellations, [10, p224].

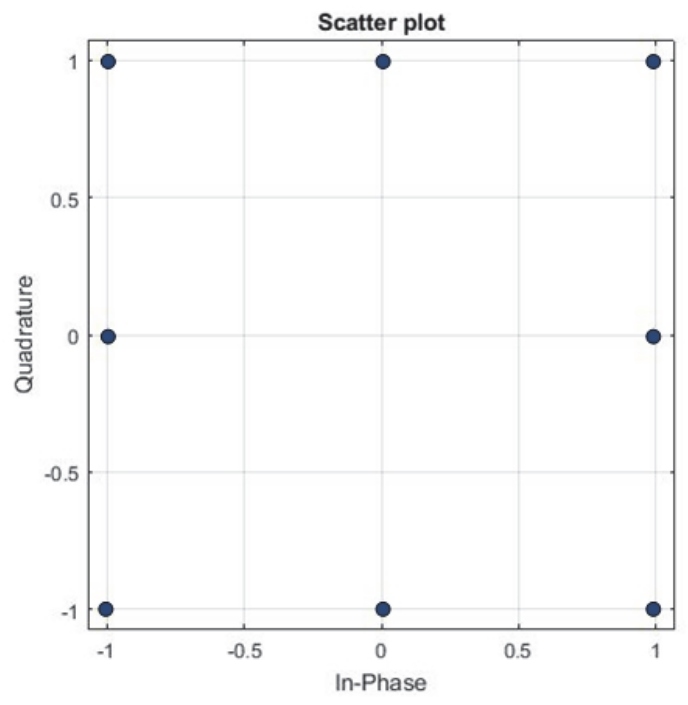

Fig. 2. Rectangular QAM-8 constellation.

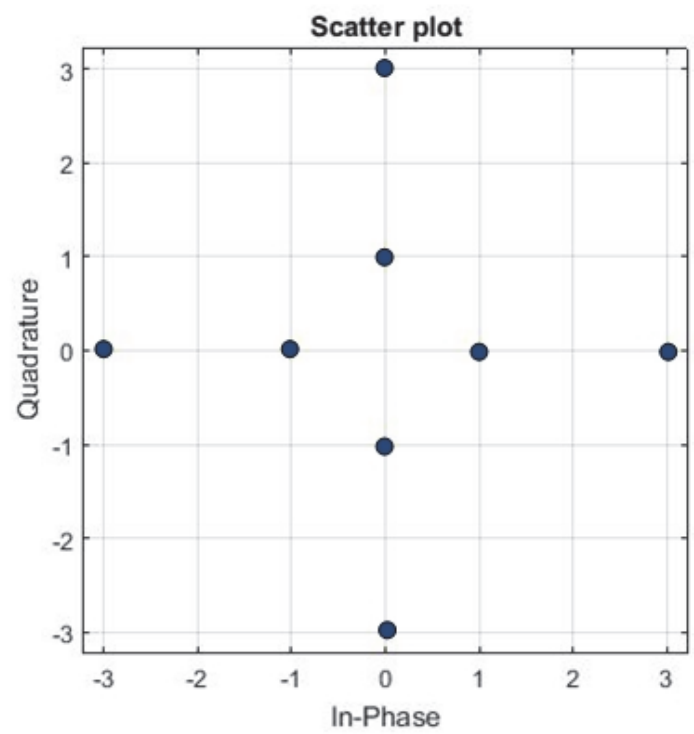

Fig. 3. Star QAM-8 constellation.

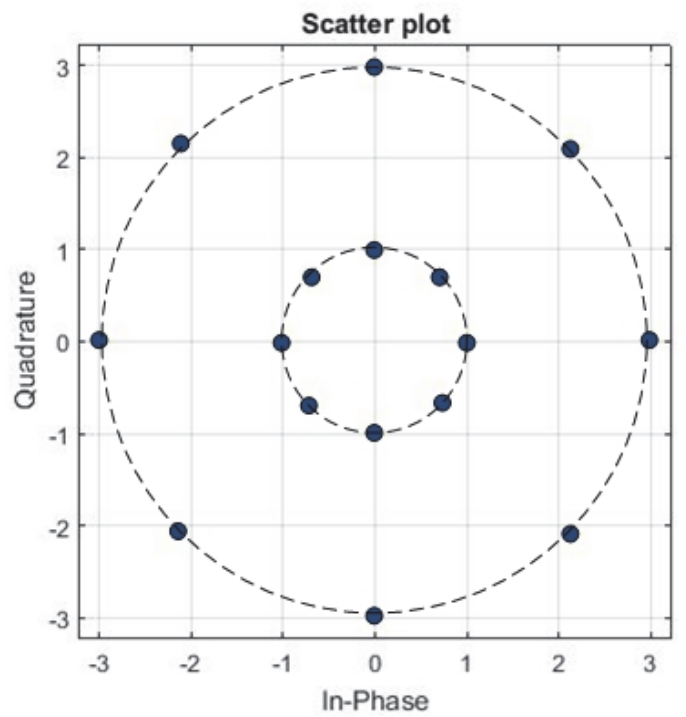

Fig. 4. Star QAM-16 constellation.

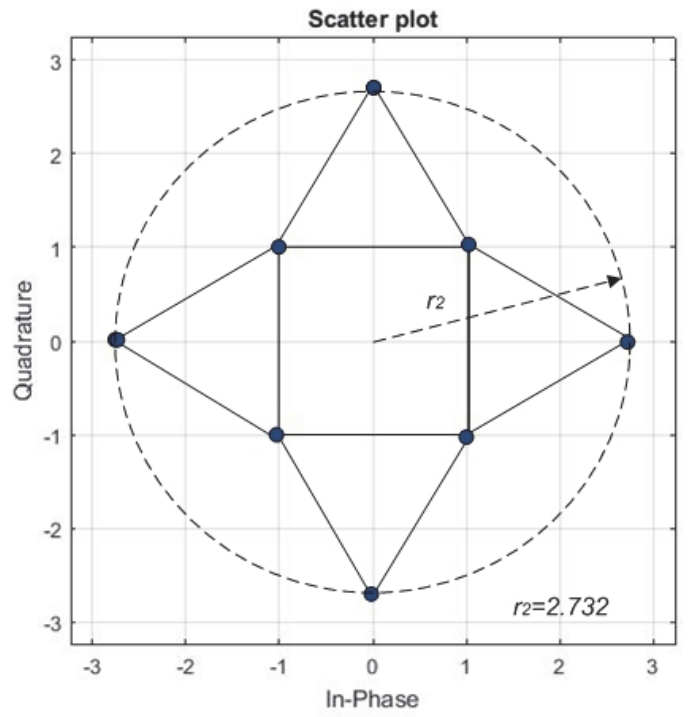

Fig. 5. Circular QAM-8 constellation.

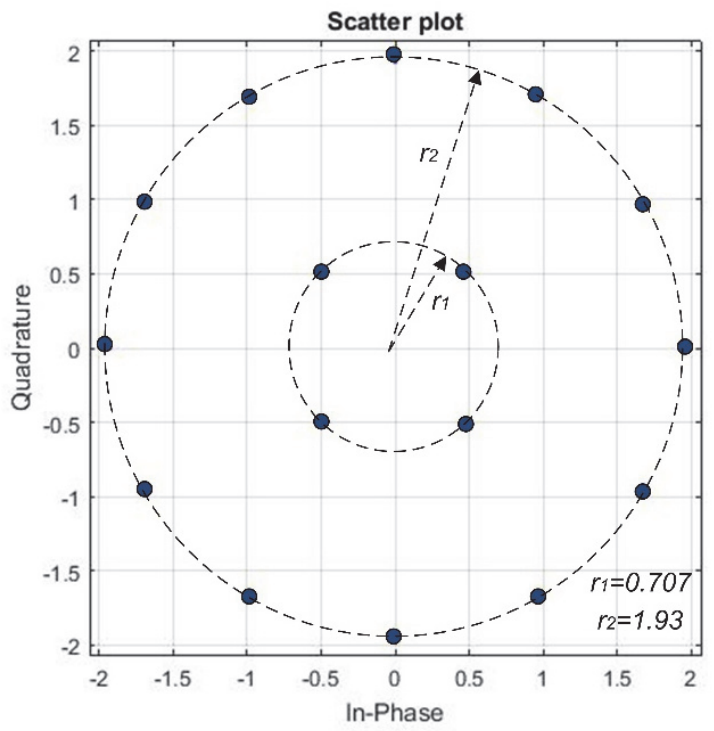

Fig. 6. (12,4) QAM-16 constellation. 


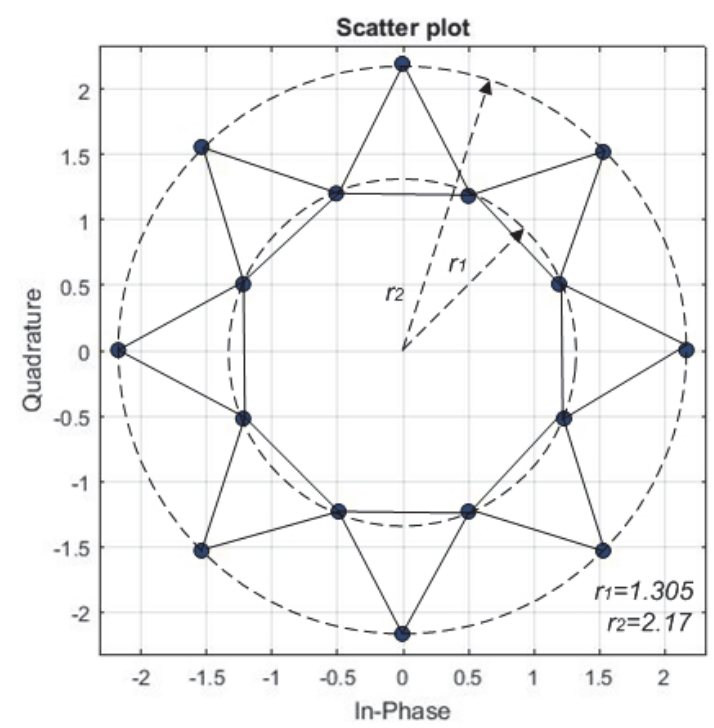

Fig. 7. (8,8) QAM-16 constellation.

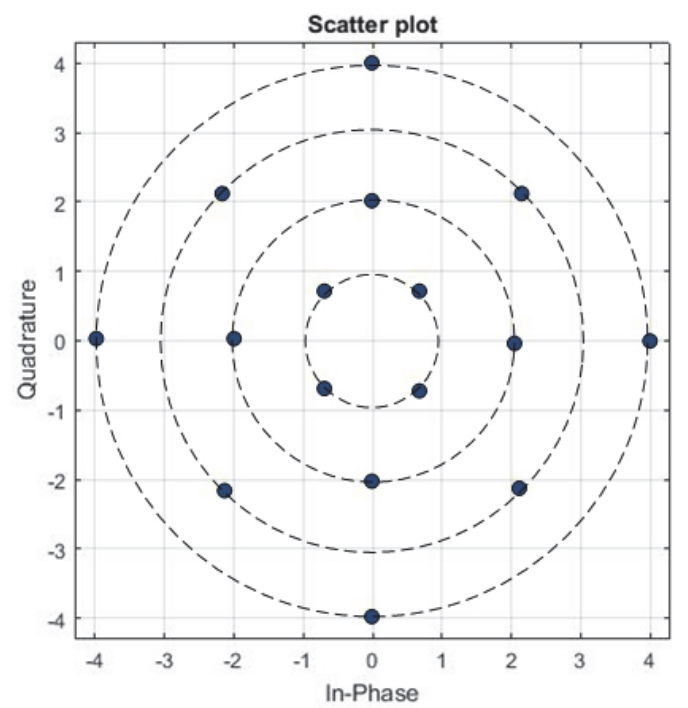

Fig. 8. $(4,4,4,4)$ QAM-16 constellation.

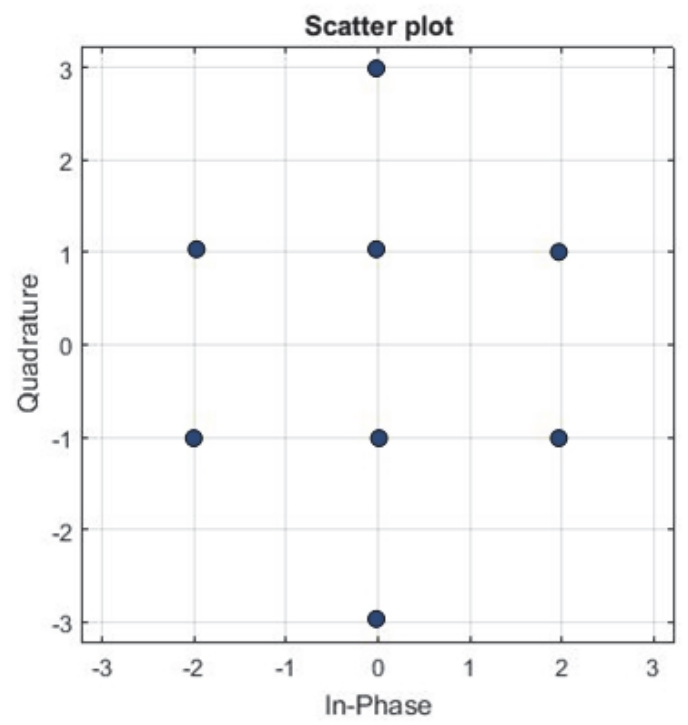

Fig. 9. AMPM-8 constellation.

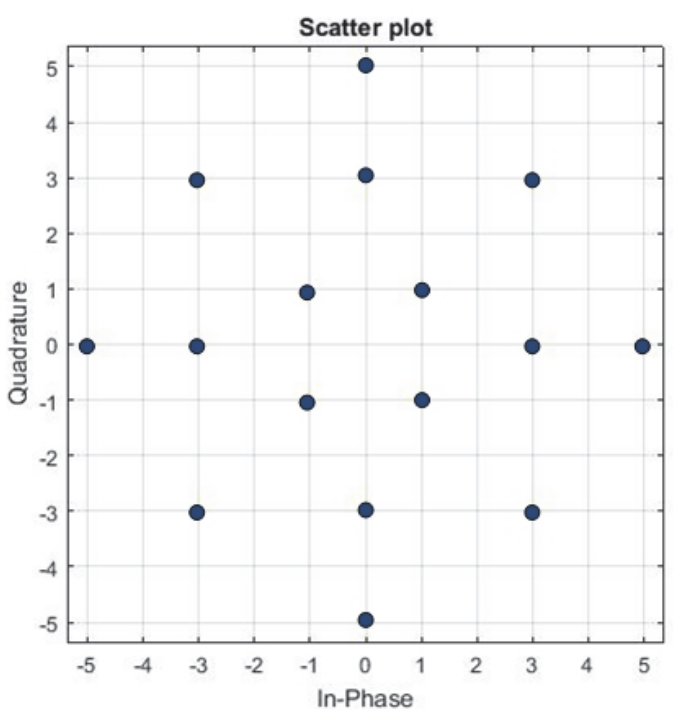

Fig. 10. V.29 constellation.

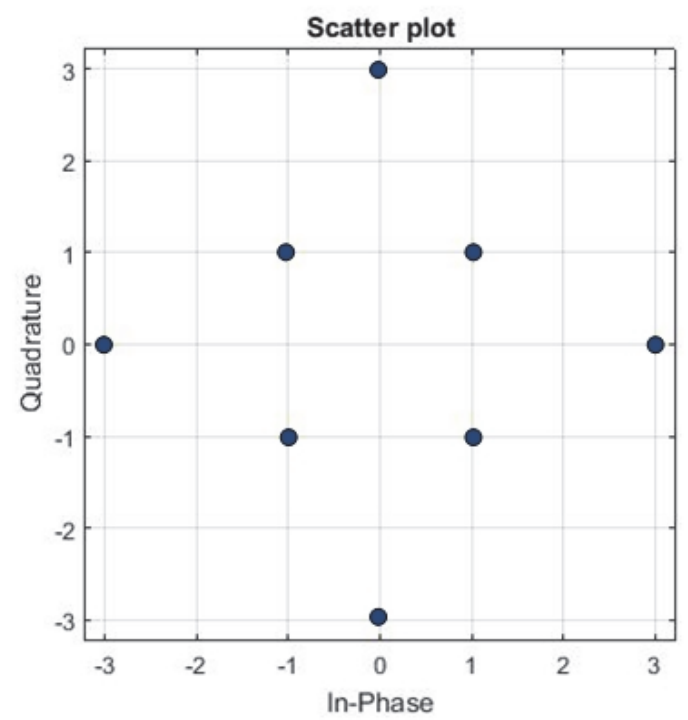

Fig. 11. V.29c (7200bps standard) constellation.

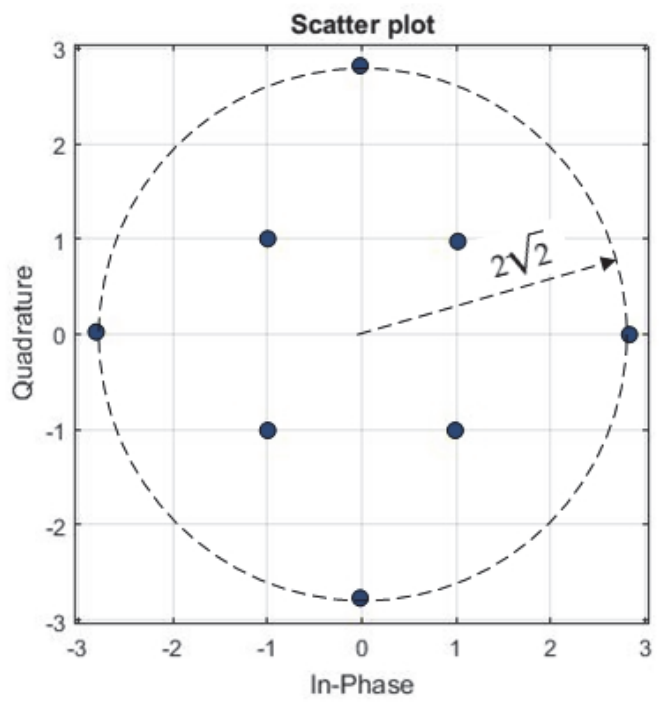

Fig. 12. V.29c in [1] constellation. 
Classical V.29, 16-point standard for $9600 \mathrm{bps}$ data transmission over a 4-wire telephone channel [15], is given in Fig. 10. Its 8-point version, standard for 7200 bps communication modem within the same recommendation [15], is given in Fig. 11 and presented under label "V29c (7200s)" in Table 1. It is interesting to note that in [1] under the label V.29c somewhere different, non-standard constellation structure is used, differing from the standard one just slightly in terms of the amplitude of four symbols. Still, this small difference showed to be sufficient for resulting with completely different higher-order cumulants' statistics, as noted in Table 1. For the reasons of comparison, 8-point V.29 constellation from [1] is also included in this analysis, presented in Fig. 12 and Table 1 under the label "V29c [1]". For comparison with other sources addressing complex signal constellations, it should be also noted that rectangular QAM-32 corresponds with V.32 standard [16] signal, while rectangular QAM-128 also corresponds with V.33 (i.e. V.32bis) standard signal for $14400 \mathrm{bps}$ communication over a telephone network channel [17]. These constellations can be found under their alternative names in literature as well (in [1], for example).

\section{AMC PERFORMANCE}

Classification performance of higher-order normalized cumulants is explained through the numerical values of error variances from eq. (6) - (9) (corresponding with particular signal constellations), along with numerical values of distance between particular higher - order cumulants. As described in [3], the ratio $\rho$ of standard deviation $\sigma$ (i.e. square root of $\operatorname{var}\left(C_{63, x}\right)$, for AMC based on sixth-order cumulants, or square root of $\operatorname{var}\left(C_{42, x}\right)$, for fourth-order cumulants) and the distance $d$ between $\hat{C}_{63}$ (i.e. $\hat{C}_{42}$ for fourth-order cumulants) values, given in Table 1 , is used to describe the efficiency of an algorithm in distinguishing one signal constellation from another. For example, when distinguishing standard V.29 from rectangular QAM-16, difference between their $\hat{C}_{63}$ values (1.4897 and 2.08, respectively) equals $d=0.5903$; standard deviations calculated from $\operatorname{var}\left(C_{63, x}\right)$ are 16.20 and 18.22, respectively, leading to the numerical value of the ratio of standard deviation and the normalized cumulant distance equal to $\rho=27.44$ for V.29 and $\rho=30.86$ for QAM-16, respectively. The same analysis for the fourth-order cumulants shows that difference between $\hat{C}_{42}$ values (0.5816 and -0.68 , respectively) equals $d=0.0984$; standard deviations calculated from $\operatorname{var}\left(C_{42, x}\right)$ are 2.96 and 3.09, leading to the numerical value of $\rho=30.08$ for V.29 and $\rho=31.40$ for QAM-16. Lower values of $\rho$ achieved for the sixth-order cumulants mean that a better classification performance in distinguishing V.29 from rectangular QAM-16 signals is expected with the sixth-order cumulants. Some examples of expected performance in distinguishing between complex constellations having the same number of symbols, by using normalized higher-order cumulants, are given in Table 2. In order to confirm expectations resulting from the numerical values of statistical parameters, a test of AMC is executed in conditions of AWGN channel, where the received signal sequence $y(n)$ can be represented by:

$$
y(n)=x(n)+g(n)
$$

with $x(n)$ standing for transmitted symbols of an unknown constellation, and $g(n)$ representing AWGN with a zero mean and variance of $\sigma_{g}^{2}$. For a zero-mean random variable $x$, associated with a transmitted data sequence $x(n)$, and a random variable $y$ associated with a received sequence $y(n)$, normalized sixth- and fourth-order cumulants can be expressed in the following manner:

$$
\begin{aligned}
& \hat{C}_{63, x}=\frac{C_{63, y}}{\left(C_{21, y}-\sigma_{g}^{2}\right)^{3}}, \\
& \hat{C}_{42, x}=\frac{C_{42, y}}{\left(C_{21, y}-\sigma_{g}^{2}\right)^{2}} .
\end{aligned}
$$

More rigorous communication channel conditions might include the presence of multipath fading, interference and other, apart from AWGN only [18].

TABLE 2: EXAMPLES OF EXPECTED AMC PERFORMANCE ESTIMATION AND COMPARISON.

\begin{tabular}{l|c|c|c|c|c}
\hline Scenario & & \multicolumn{2}{|c|}{$\hat{C}_{63}$} & \multicolumn{2}{|c}{$\hat{C}_{42}$} \\
\hline \multirow{3}{*}{ V.29 vs rQAM-16 } & $d$ & 0.5903 & \multicolumn{2}{|c}{0.0984} \\
& $\sigma$ & 16.20 & 18.22 & 2.96 & 3.09 \\
& $\rho=\sigma / d$ & $\mathbf{2 7 . 4 4}$ & $\mathbf{3 0 . 8 6}$ & 30.08 & 31.40 \\
& & $d$ & 3.1700 & \multicolumn{2}{|c|}{0.5300} \\
sQAM-8 vs rQAM-8 & $\sigma$ & 10.81 & 22.04 & 2.62 & 3.33 \\
& $\rho=\sigma / d$ & $\mathbf{3 . 4 1}$ & 6.95 & 4.94 & $\mathbf{6 . 2 8}$ \\
\hline
\end{tabular}

We carried out the simulations through 2000 Monte Carlo trials and $N=2000$ received data samples were collected for AMC in each trial, where algorithms based on $\hat{C}_{63}$ and $\hat{C}_{42}$ values were tested in an AWGN channel, in scenario with modulation candidates considered from the set: $\{$ V.29, QAM-16 . The value of $N$ was selected to match directly with the one used in simulations for complex constellations described in [3]. The AWGN channel was simulated with noise power $\sigma_{g}^{2}$ considered to be known. Correct classification probability $P_{C C}$ was calculated versus $S N R$, and Fig. 13 illustrates the results of simulation.

Results presented in Fig. 13 confirm the expectation of theoretical analysis: classification performance in considered scenario is slightly better with the sixth-order cumulants, indeed. Additionally, one interesting property of $\hat{C}_{63}$ explained in [7], the presence of bias for PAM signals, which improves the classification of these signals significantly in comparison with $\hat{C}_{42}$ - based AMC, can be verified in an experiment with an expanded set of constellation candidates. Again, simulations through 2000 Monte Carlo trials were carried out, with both algorithms tested in AWGN channel conditions, this time in scenario with modulation candidates from the set: \{V.29, QAM-16, PAM-16\}, and results are presented in Fig. 14. 


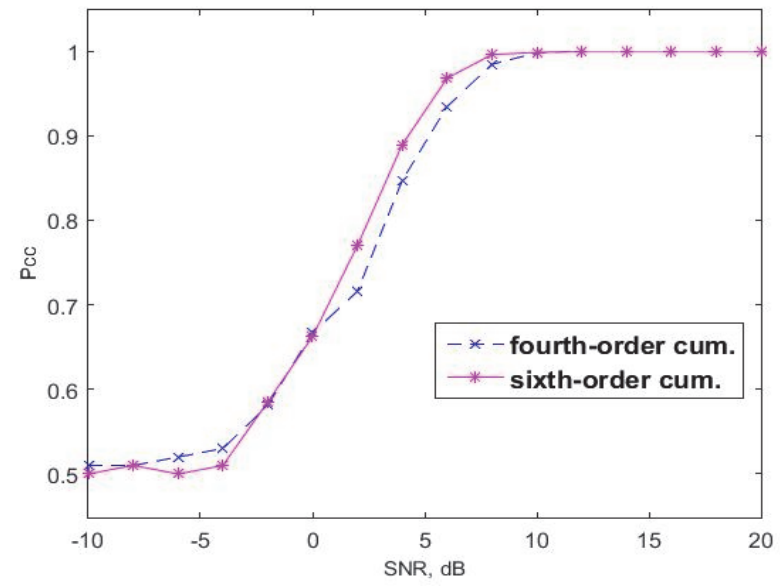

Fig. 13. Correct classification probability in $\{$ V.29, QAM-16 $\}$ scenario, $N=2000$, AWGN channel.

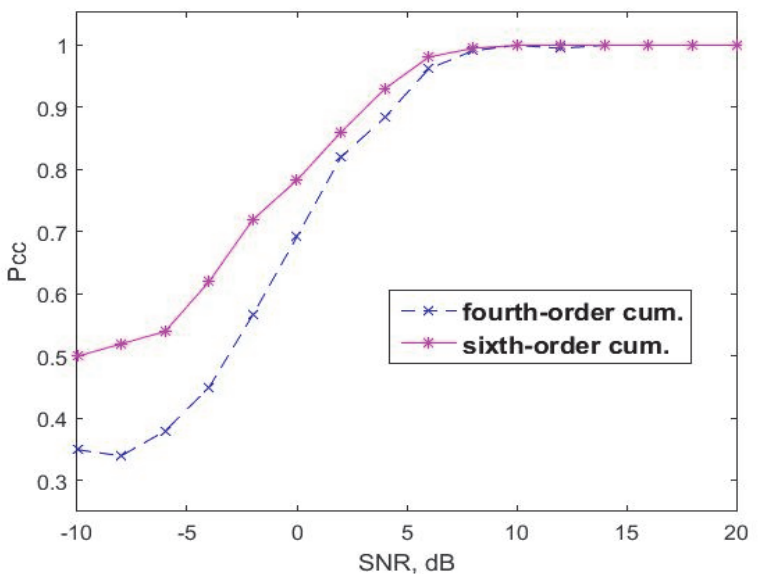

Fig. 14. Correct classification probability in $\{$ V.29, QAM-16, PAM-16 $\}$ scenario, $N=2000$, AWGN channel.

From Fig. 14 it can be concluded that a very good performance in distinguishing real from complex constellations with the sixth-order cumulants leads to a more significant difference in achieved $P_{C C}$ even in the case with a wider set of candidates from the class of complex constellations. Errorless classification of PAM-16 signals with $\hat{C}_{63}$ algorithm at all $S N R$ values higher than $4 \mathrm{~dB}$ directly leads to its superior performance over the fourth-order cumulants, as illustrated in Fig. 14, being also clearly much more dominant than in the case when complex signals only are classified (Fig. 13). A similar analysis can be carried out for any constellation given in this paper: presented statistics provide a possibility for estimation of expected classification performance on the basis of ratio $\rho$, in procedure as demonstrated.

\section{CONCLUSION}

In this paper AMC statistics for a number of complex constellations are presented for the first time, classification performance is discussed and tested via simulations. While simulations show a good level of alignment with theoretical expectations, precise visual and numerical description of many constellation shapes, presented in cumulant-based concept of $\mathrm{AMC}$, do provide necessary repeatability, and resolve any potential ambiguities in numerical values and interpretation thereof. In future work, it will be of interest to explore the benefits of recursive modulation order reduction over the rectangular QAM constellations with a huge number of symbols, as potentially additional AMC performance accelerator.

\section{REFERENCES}

[1] A. Swami and B. M. Sadler, "Hierarchical Digital Modulation Classification Using Cumulants," IEEE Trans. Commun., Vol. 48, pp. 416-429, 2000.

[2] C. M. Spooner, "On the utility of sixth-order cyclic cumulants for RF signal classification," In Conference Record of Thirty-Fifth Asilomar Conference on Signals, Systems and Computers (Cat. No. 01CH37256), vol. 1, pp. 890-897. IEEE, 2001.

[3] V. D. Orlic and M. L. Dukic, "Automatic modulation classification algorithm using higher-order cumulants under real-world channel conditions," IEEE Communications Letters 13, no. 12 (2009): 917 919.

[4] O. A. Dobre, A. Abdi, Y. Bar-Ness, and W. Su, "Survey of automatic modulation classification techniques: classical approaches and new trends," IET communications 1, no. 2 (2007): 137-156.

[5] M. S. Pajic, M. Veinovic, M. Peric, and V. D. Orlic. "Modulation Order Reduction Method for Improving the Performance of AMC Algorithm Based on Sixth-Order Cumulants," IEEE Access 8 (2020): 106386-106394.

[6] V. D. Orlic and M. L. Dukic, "Properties of an Algorithm for Automatic Modulation Classification Based on Sixth-Order Cumulants." In Proceedings of the XLIV ICEST 2009 Conference, Veliko Tarnovo, Bulgaria, 2009, vol. 2, p. 635-638.

[7] M. Simic, M. Stankovic, V. D. Orlic, "Automatic Modulation Classification of Real Signals in AWGN Channel Based on SixthOrder Cumulants," Radioengineering Journal, Vol. 30, No. 1, April 2021, pp. 204-214.

[8] O. A. Dobre, M. Oner, S. Rajan and R. Inkol, "Cyclostationaritybased robust algorithms for QAM signal identification," IEEE Comm. Letters 16, no. 1 (2011): 12-15.

[9] C. M. Spooner, A. N. Mody, J. Chuang and J. Petersen, "Modulation recognition using second-and higher-order cyclostationarity," In 2017 IEEE Int. Symp. on Dynamic Spectrum Access Networks (DySPAN), pp. 1-3. IEEE, 2017.

[10] S. Benedetto, E. Biglieri, and V. Castellani, Digital Transmission Theory. Englewood Cliffs, New Jersey: Prentice Hall, 1987.

[11] Y.-C. Lin, and C-C. Jay Kuo, "Classification of quadrature amplitude modulated (QAM) signals via sequential probability ratio test (SPRT)," Signal Processing 60, no. 3 (1997): 263-280.

[12] X. Dong, Coherent signalling and receiver diversity for fading channels. Queen's University, 2000.

[13] C. Thomas, M. Weidner and S. Durrani, "Digital amplitude-phase keying with M-ary alphabets," IEEE Transactions on Communications 22, no. 2 (1974): 168-180.

[14] J. M. Cioffi, "Discrete Data Transmission Basics - Part I: Modulation and Canonical Reception", Chapters for EE379A: Digital Communications - Signal Processing, available online: https://cioffi-group.stanford.edu/doc/book/chap1.pdf, Visited on 22.04.2021.

[15] ITU-T Recommendation V.29: Data Communication Over the Telephone Network - 9600 bits per second Modem Standardized for use on Point-to-Point 4-Wire Leased Telephone-Type Circuits, Recommendation V.29, Int. Telecomm. Union ITU-T, 1993.

[16] ITU-T Recommendation V.32: A family of 2-wire, duplex modems operating at data rates of up to $9600 \mathrm{bit} / \mathrm{s}$ for use on the general switched telephone network and leased telephone-type circuits, Recommendation V.32, Int. Telecomm. Union ITU-T, 1993.

[17] CCITT Recommendation V.32 bis: A duplex modem operating at data signaling rates of up to $14400 \mathrm{bit} / \mathrm{s}$ for use on the general switched telephone network and on leased point-to-point 2-wire telephone-type circuits, Recommendation V.32 bis, Consultative Committee for International Telegraphy and Telephony CCITT International Telecommunication Union ITU-T, 1991.

[18] V. D. Orlic and M. L. Dukic, "Automatic modulation classification: Sixth-order cumulant features as a solution for real-world challenges," In 2012 20th Telecommunications Forum (TELFOR), pp. 392-399. IEEE, 2012. 